\title{
Homocoupling of aryl halides in flow: Space integration of lithiation and $\mathrm{FeCl}_{3}$ promoted homocoupling
}

\author{
Aiichiro Nagaki, Yuki Uesugi, Yutaka Tomida and Jun-ichi Yoshida*
}

\section{Full Research Paper}

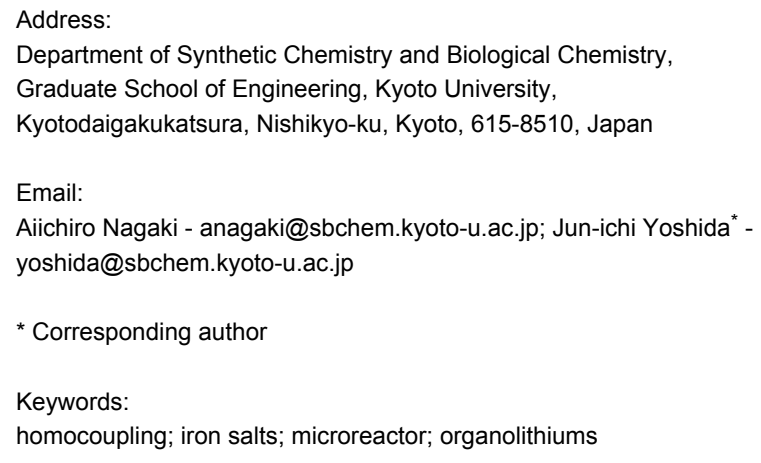

Beilstein J. Org. Chem. 2011, 7, 1064-1069. doi:10.3762/bjoc.7.122

Received: 15 April 2011

Accepted: 29 June 2011

Published: 02 August 2011

This article is part of the Thematic Series "Chemistry in flow systems II".

Guest Editor: A. Kirschning

(c) 2011 Nagaki et al; licensee Beilstein-Institut. License and terms: see end of document.

\begin{abstract}
The use of $\mathrm{FeCl}_{3}$ resulted in a fast homocoupling of aryllithiums, and this enabled its integration with the halogen-lithium exchange reaction of aryl halides in a flow microreactor. This system allows the homocoupling of two aryl halides bearing electrophilic functional groups, such as $\mathrm{CN}$ and $\mathrm{NO}_{2}$, in under a minute.
\end{abstract}

\section{Introduction}

Biaryl structures often occur in various organic compounds including natural products, bioactive compounds, functional polymers, ligands in catalysts and theoretically interesting molecules, and the oxidative homocoupling of arylmetals is one of the most useful methods for the construction of biaryl frameworks [1]. Stoichiometric amounts of transition metal salts such as $\mathrm{TiCl}_{4}$ [2], $\mathrm{TlCl}$ [3], $\mathrm{VO}(\mathrm{OEt}) \mathrm{Cl}_{2}$ [4], $\mathrm{CoCl}_{2}$ [5], $\mathrm{CuCl}_{2}$ [6] and $\mathrm{Pd}(\mathrm{OAc})_{2}$ [7] have been used for homocoupling of arylmetals. In some cases catalytic processes in the presence of a reoxidant, such as oxygen or other organic oxidants, are effective. Recently, iron salts have been also used because of their low costs and lack of toxicity [8-18]. For example, Hayashi et al. reported the iron-catalyzed oxidative homocoupling of Grignard reagents, using 1,2-dihalogenoethanes as an oxidant [19]. Cahiez et al. have also reported the $\mathrm{FeCl}_{3}$-catalyzed homocou- pling reaction of Grignard reagents bearing functional groups, using atmospheric oxygen [20]. The use of aryllithium compounds instead of Grignard reagents is very interesting, because they are easily generated by halogen-lithium exchange under homogeneous conditions, thus enabling the generation in a flow. However, to the best of our knowledge, oxidative homocoupling of aryllithiums using iron salts has not been reported so far. One of the major reasons for this seems to be the instability of aryllithiums, especially of those bearing electrophilic functional groups such as cyano and nitro groups [21], making the subsequent homocoupling difficult or impossible.

Recently, we have reported that flow microreactor systems [2285 ] are quite effective for the generation and reaction of highly reactive organolithiums such as functionalized aryllithiums, 
oxiranyllithums, aziridinyllithiums, and allenyllithiums [86-98]. Herein we report that integration $[99,100]$ of the generation of aryllithiums, especially those bearing electrophilic functional groups, by halogen-lithium exchange and $\mathrm{FeCl}_{3}$ promoted homocoupling has been effectively accomplished in an integrated flow microreactor system.

\section{Results and Discussion}

First, we focused on the generation of $p$-methoxyphenyllithium from $p$-bromoanisole (Scheme 1). A flow microreactor system, consisting of two T-shaped micromixers (M1 and M2) and two microtube reactors (R1 and R2) shown in Figure 1, was used. A solution of $p$-bromoanisole (Ar-X) ( $0.10 \mathrm{M}$ in THF, flow rate: $6.0 \mathrm{~mL} / \mathrm{min})$ and a solution of $n$-butyllithium $(0.40 \mathrm{M}$ in hexane, flow rate: $1.5 \mathrm{~mL} / \mathrm{min}$ ) were introduced to $\mathbf{M 1}(\varnothing=$ $250 \mu \mathrm{m})$ by syringe pumps. The resulting mixture was passed through $\mathbf{R} 1$ to conduct the bromine-lithium exchange reaction. Methanol (neat, flow rate: $3.0 \mathrm{~mL} / \mathrm{min}$ ) was added in $\mathbf{M 2}(\varnothing=$ $500 \mu \mathrm{m})$ and the mixture was passed through $\mathbf{R 2}(\varnothing=1000$ $\mu \mathrm{m}, \mathrm{L}=50 \mathrm{~cm}$ ) to protonate $p$-methoxyphenyllithium. The reactions were carried out with varying residence time in $\mathbf{R} \mathbf{1}$ $\left(t^{\mathrm{R} 1}: 0.2-6.3 \mathrm{~s}\right)$ and varying temperature $\left(T:-78\right.$ to $\left.24^{\circ} \mathrm{C}\right)$. The temperature $(T)$ was controlled by adjusting the bath temperature. The residence time $\left(t^{\mathrm{R} 1}\right)$ was adjusted by changing the inner diameter and the length in the microtube reactor $\mathbf{R} \mathbf{1}$ with a fixed flow rate. After a steady state was reached, the product solution was collected for $30 \mathrm{~s}$. As shown in Figure 2, the yield of the protonated product, anisole, depends on both $T$ and $t^{\mathrm{R} 1}$. The reaction at low temperatures $\left(T<-48{ }^{\circ} \mathrm{C}\right)$ with short residence times $\left(t^{\mathrm{R} 1}<0.79 \mathrm{~s}\right)$ resulted in very low yields, because the $\mathrm{Br}-\mathrm{Li}$ exchange reaction was not complete. The increase in $T$ and $t^{\mathrm{R} 1}$ caused an increase in the yield, and high yields $(>85 \%)$ were obtained through the appropriate choice of $T$ and $t^{\mathrm{R} 1}$.

Next, we examined the integration of the halogen-lithium exchange reaction with $\mathrm{FeCl}_{3}$ promoted homocoupling (Scheme 2). Integrated flow microreactor systems consisting of three micromixers (M1, M2, and M3) and three microtube reac-

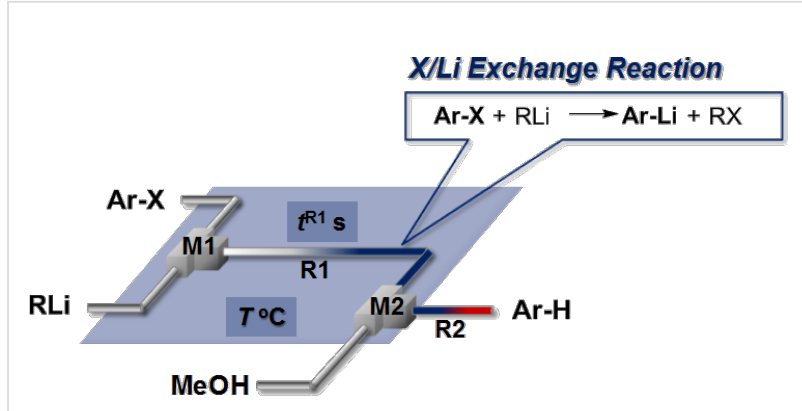

Figure 1: Flow microreactor system for halogen-lithium exchange of aryl halide followed by reaction with methanol. T-shaped micromixer: M1 (inner diameter: $250 \mu \mathrm{m}$ ), and M2 (inner diameter: $500 \mu \mathrm{m}$ ), microtube reactor: $\mathbf{R} \mathbf{1}$ and $\mathbf{R} \mathbf{2}(\varnothing=1000 \mu \mathrm{m}$, length $=50 \mathrm{~cm})$, a solution of aryl halides: $0.10 \mathrm{M}$ in THF $(6.0 \mathrm{~mL} / \mathrm{min})$, a solution of lithium reagent: $0.40 \mathrm{M}$ or $0.42 \mathrm{M}$ in hexane ( $n-\mathrm{BuLi})$ or $\mathrm{Et}_{2} \mathrm{O}(\mathrm{PhLi})(1.5 \mathrm{~mL} / \mathrm{min}), \mathrm{a}$ solution of methanol: Neat $(3.0 \mathrm{~mL} / \mathrm{min})$.

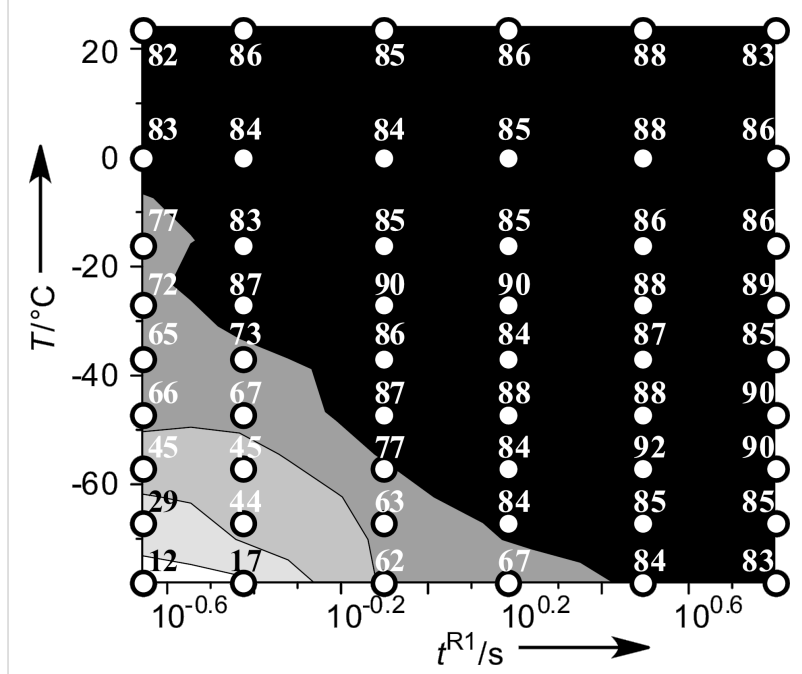

Figure 2: Effects of the temperature $(T)$ and the residence time in $\mathbf{R 1}$ $\left(t^{R 1}\right)$ on the yield of anisole in the $\mathrm{Br}$-Li exchange reaction of $p$-bromoanisole followed by reaction with methanol in the flow microreactor system. The contour plot with scatter overlay shows the yields of anisole $(\%)$, which are indicated by small circles.

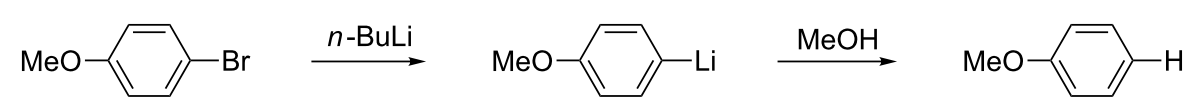

Scheme 1: Halogen-lithium exchange of $p$-bromoanisole followed by reaction with methanol.

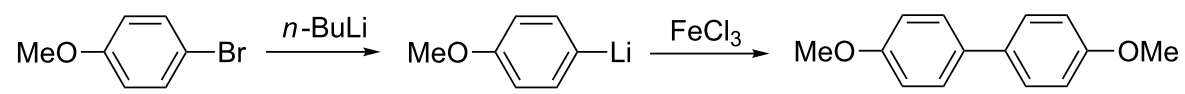


tors ( $\mathbf{R 1}, \mathbf{R 2}$, and $\mathbf{R 3})$ were used, as shown in Figure 3. A solution of $p$-bromoanisole $(\mathrm{Ar}-\mathrm{X})(0.10 \mathrm{M}$ in THF, flow rate: 6.0 $\mathrm{mL} / \mathrm{min})$ and a solution of $n$-butyllithium $(0.42 \mathrm{M}$ in hexane, flow rate: $1.5 \mathrm{~mL} / \mathrm{min})$ were introduced to $\mathbf{M 1}(\varnothing=250 \mu \mathrm{m})$ by syringe pumps. The resulting mixture was passed through $\mathbf{R} 1\left(t^{\mathrm{R} 1}=13 \mathrm{~s}\left(-78{ }^{\circ} \mathrm{C}\right), t^{\mathrm{R} 1}=13 \mathrm{~s}\left(-48^{\circ} \mathrm{C}\right), t^{\mathrm{R} 1}=3.1 \mathrm{~s}(-28\right.$ $\left.\left.{ }^{\circ} \mathrm{C}\right), t^{\mathrm{R} 1}=3.1 \mathrm{~s}\left(0^{\circ} \mathrm{C}\right), t^{\mathrm{R} 1}=3.1 \mathrm{~s}\left(24^{\circ} \mathrm{C}\right)\right)$ at the corresponding temperatures and was mixed with a solution of $\mathrm{FeCl}_{3}(0.10 \mathrm{M}$ in THF, flow rate: $6.0 \mathrm{~mL} / \mathrm{min})$ in $\mathbf{M} 2(\varnothing=500 \mu \mathrm{m})$. The resulting mixture was passed through $\mathbf{R 2}$ and was then mixed with methanol (neat, flow rate: $1.5 \mathrm{~mL} / \mathrm{min})$ in $\mathbf{M 3}(\varnothing=500$ $\mu \mathrm{m})$ to protonate the unchanged $p$-methoxyphenyllithium. The resulting solution was passed through $\mathbf{R} 3(\varnothing=1000 \mu \mathrm{m}, \mathrm{L}=$ $50 \mathrm{~cm})$. The temperature $(T)$ was controlled by adjusting the bath temperature, and the residence time in $\mathbf{R} 2\left(t^{\mathrm{R} 2}\right)$ by changing the inner diameter and the length in $\mathbf{R} \mathbf{2}$ with the fixed flow rate. After a steady state was reached, the product solution was collected for $30 \mathrm{~s}$. The results obtained from varying $t^{\mathrm{R} 2}$ and $T$ are summarized in Figure 4, in which the yield of 4,4'dimethoxybiphenyl is plotted against $T$ and $t^{\mathrm{R} 2}$ as a contour map with scattered overlay (see Supporting Information File 1 for details). The yield depends on both $T$ and $t^{\mathrm{R} 2}$. At $-78^{\circ} \mathrm{C}$, the yield increased with $t^{\mathrm{R} 2}$ because of the progress of the homocoupling. At $0{ }^{\circ} \mathrm{C}$, the homocoupling product was obtained in reasonable yields for a wide range of $t^{\mathrm{R} 2}$. The productivity of the present system is acceptable for large scale laboratory synthesis $(6.2 \mathrm{~g} / \mathrm{h})$. It is noteworthy that the integrated reactions were complete within the overall residence time of $14.7 \mathrm{~s}$, even at low temperatures such as $-48{ }^{\circ} \mathrm{C}$. Thus, we envisaged that the reaction could also be applied to less stable aryllithium compounds that decompose very quickly.

One of the major benefits of flow microreactor synthesis is the ability to use highly unstable reactive intermediates. Such inter-

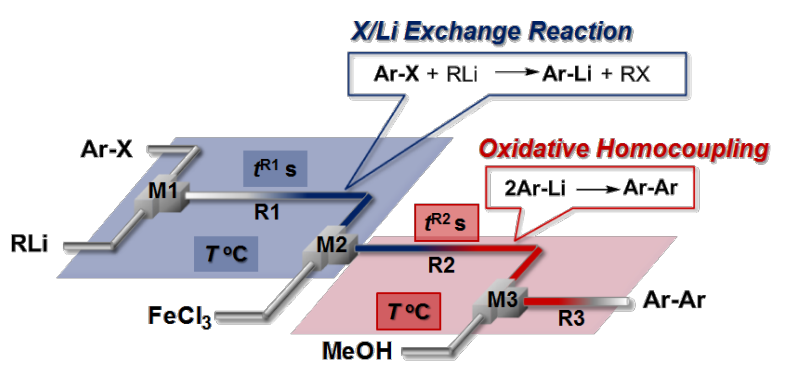

Figure 3: Integrated flow microreactor system for oxidative homocoupling reaction of aryllithium with $\mathrm{FeCl}_{3}$. T-shaped micromixer: M1 (inner diameter: $250 \mu \mathrm{m}$ ), M2 (inner diameter: $500 \mu \mathrm{m}$ ), and M3 (inner diameter: $500 \mu \mathrm{m})$, microtube reactor: $\mathbf{R} 1, \mathbf{R} 2$ and $\mathbf{R} 3(\varnothing=1000 \mu \mathrm{m}$, length $=50 \mathrm{~cm})$, a solution of aryl halides: $0.10 \mathrm{M}$ in THF $(6.0 \mathrm{~mL} / \mathrm{min})$, a solution of lithium reagent: $0.42 \mathrm{M}$ in hexane $(n-\mathrm{BuLi})$ or $\mathrm{Et}_{2} \mathrm{O}(\mathrm{PhLi})$ $(1.5 \mathrm{~mL} / \mathrm{min})$, a solution of $\mathrm{FeCl}_{3}: 0.10 \mathrm{M}$ in THF $(6 \mathrm{~mL} / \mathrm{min})$, a solution of methanol: Neat $(1.5 \mathrm{~mL} / \mathrm{min})$.

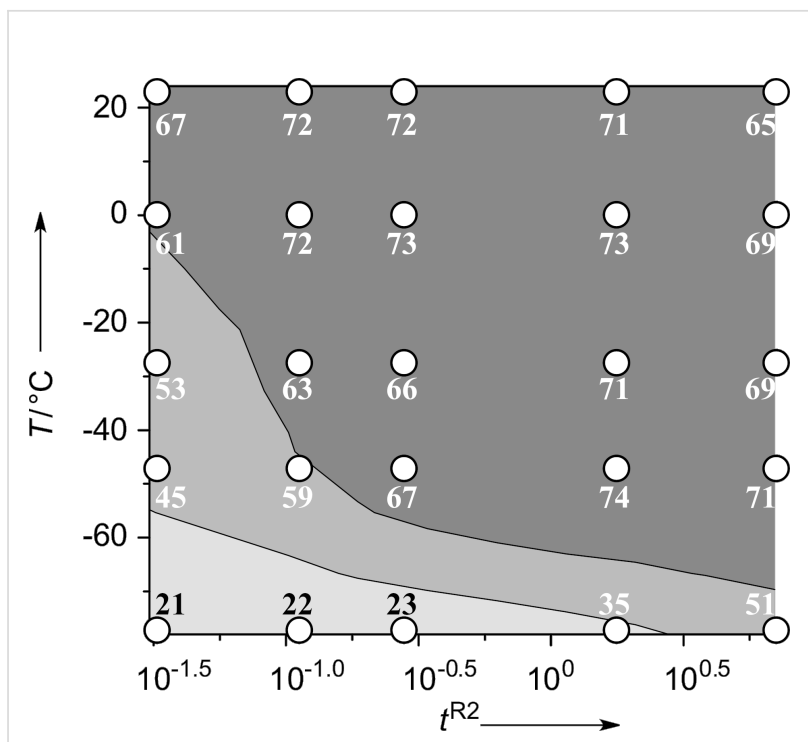

Figure 4: Effects of the temperature $(T)$ and the residence time in $\mathbf{R 2}$ $\left(t^{\mathrm{R} 2}\right)$ on the yield of $4,4^{\prime}$-dimethoxybiphenyl in the oxidative homocoupling of $p$-methoxyphenyllithium with $\mathrm{FeCl}_{3}$ in the integrated flow microreactor system. Contour plot with scatter overlay of the yields of 4,4'-dimethoxybiphenyl (\%), which are indicated by small circles.

mediates can be rapidly generated and transferred to another location to be used in a subsequent reaction before they decompose. We have already reported the generation and reactions of unstable aryllithium species such as $o$-bromophenyllithiums, and aryllithiums bearing alkoxycarbonyl, cyano, nitro, and ketone carbonyl groups $[86,87,93,95,96,98]$, which are difficult to use in conventional macro batch reactors. As shown in Table 1, reactions of aryllithiums bearing cyano and nitro groups proceeded successfully to give the corresponding homocoupling products, where in contrast it is very difficult to achieve such reactions using conventional batch reactors. A mechanism involving transmetalation of the aryl group from lithium to iron followed by reductive elimination of the homocoupling product seems to be plausible, while a similar mechanism is proposed for homo-coupling of organomagnesium compounds with $\mathrm{FeCl}_{3}[19,20]$. The regiospecificity of the coupling is consistent with this mechanism. Radical coupling seems to be less likely.

\section{Conclusion}

In conclusion, we found that the use of $\mathrm{FeCl}_{3}$ results in fast oxidative homocoupling of aryllithiums, which enables its integration with the halogen-lithium exchange of aryl halides. Various aryl halides, including those bearing electrophilic functional groups, can be used for this transformation in the integrated flow microreactor system. Hence, the method greatly enhances the synthetic utility of aryllithium compounds and adds a new dimension to the chemistry of coupling reactions. 
Table 1: Homocoupling of aryl halides using the integrated flow microreactor system.

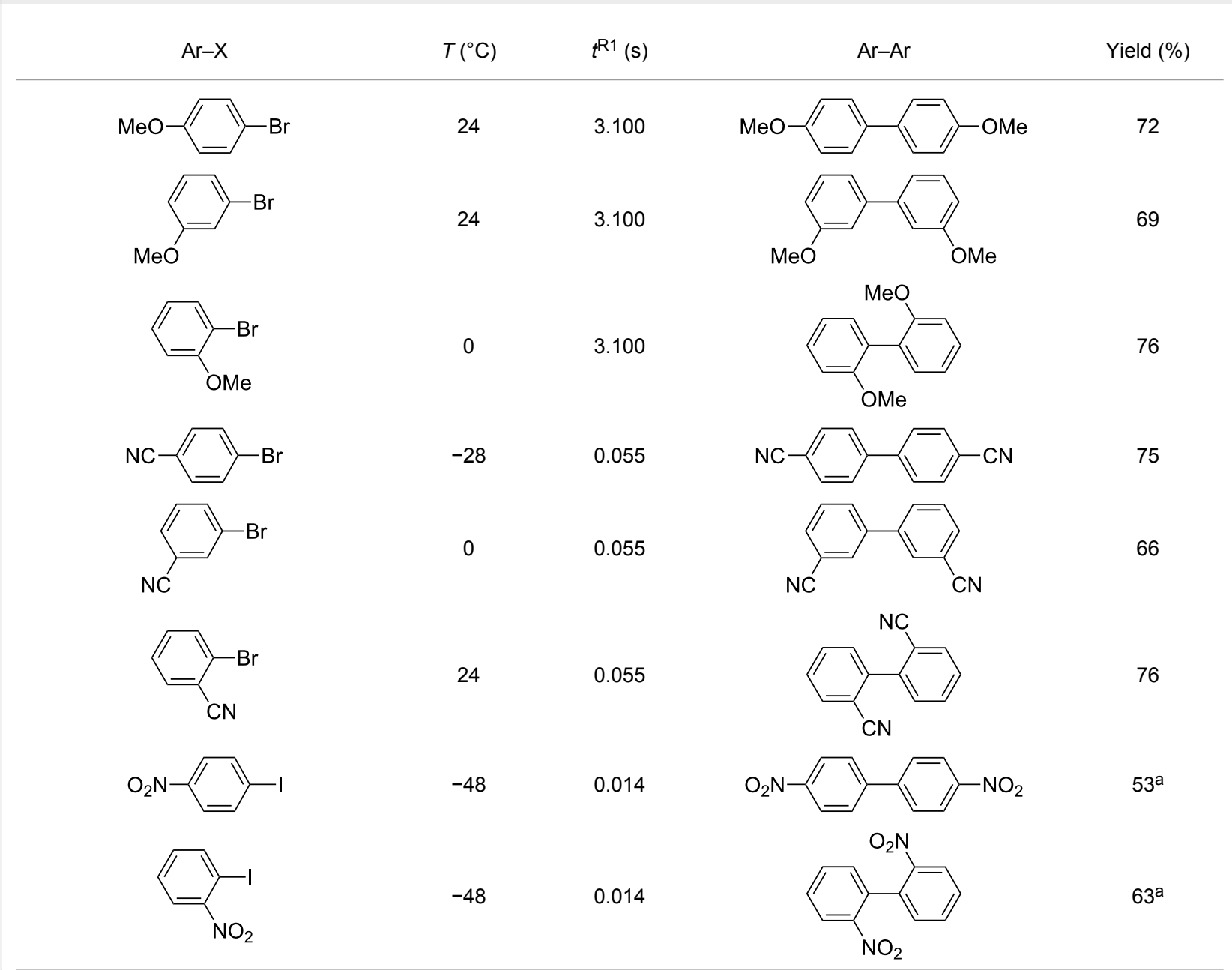

aphLi instead of $n$-BuLi was used as lithiating reagent.

\section{Supporting Information}

Supporting Information features experimental procedures and full spectroscopic data for all new compounds.

\section{Supporting Information File 1}

Experimental details.

[http://www.beilstein-journals.org/bjoc/content/ supplementary/1860-5397-7-122-S1.pdf]

\section{Acknowledgements}

This work was financially supported in part by a Grant-in-Aid for Scientific Research from the Japan Society for the Promotion of Science and NEDO projects.

\section{References}

1. Cepanec, I. Synthesis of Biaryls; Elsevier: Amsterdam, Boston, 2004.

2. Inoue, A.; Kitagawa, K.; Shinokubo, H.; Oshima, K. Tetrahedron 2000, 56, 9601-9605. doi:10.1016/S0040-4020(00)00929-7

3. McKillop, A.; Elsom, L. F.; Taylor, E. C. J. Am. Chem. Soc. 1968, 90, 2423-2424. doi:10.1021/ja01011a041

4. Ishikawa, T.; Ogawa, A.; Hirao, T. Organometallics 1998, 17, 5713-5716. doi:10.1021/om980607c

5. Kharasch, M. S.; Fields, E. K. J. Am. Chem. Soc. 1941, 63, 2316-2320. doi:10.1021/ja01854a006

6. Sakellarios, E.; Kyrimis, T. Ber. Dtsch. Chem. Ges. 1924, 57, 322-326. doi:10.1002/cber.19240570233

7. Lei, A.; Srivastava, M.; Zhang, X. J. Org. Chem. 2002, 67, 1969-1971. doi:10.1021/jo011098i

8. Cahiez, G.; Marquais, S. Pure Appl. Chem. 1996, 68, 53-60. doi:10.1351/pac199668010053

9. Cahiez, G.; Marquais, S. Tetrahedron Lett. 1996, 37, 1773-1776. doi:10.1016/0040-4039(96)00116-5 
10. Cahiez, G.; Avedissian, H. Synthesis 1998, 1199-1205 doi:10.1055/s-1998-2135

11. Dohle, W.; Kopp, F.; Cahiez, G.; Knochel, P. Synlett 2001, 1901-1904. doi:10.1055/s-2001-18748

12. Fürstner, A.; Leitner, A.; Méndez, M.; Krause, H. J. Am. Chem. Soc. 2002, 124, 13856-13863. doi:10.1021/ja027190t

13. Fürstner, A.; Leitner, A. Angew. Chem., Int. Ed. 2002, 41, 609-612. doi:10.1002/1521-3773(20020215)41:4<609::AID-ANIE609>3.0.CO;2$\mathrm{M}$

14. Quintin, J.; Franck, X.; Hocquemiller, R.; Figadère, B. Tetrahedron Lett. 2002, 43, 3547-3549. doi:10.1016/S0040-4039(02)00568-3

15. Martin, R.; Fürstner, A. Angew. Chem., Int. Ed. 2004, 43, 3955-3957. doi:10.1002/anie.200460504

16. Scheiper, B.; Bonnekessel, M.; Krause, H.; Fürstner, A. J. Org. Chem. 2004, 69, 3943-3949. doi:10.1021/j00498866

17. Nakamura, M.; Matsuo, K.; Ito, S.; Nakamura, E. J. Am. Chem. Soc. 2004, 126, 3686-3687. doi:10.1021/ja049744t

18. Nagano, T.; Hayashi, T. Org. Lett. 2004, 6, 1297-1299. doi:10.1021/ol049779y

19. Nagano, T.; Hayashi, T. Org. Lett. 2005, 7, 491-493. doi:10.1021/ol047509+

20. Cahiez, G.; Chaboche, C.; Mahuteau-Betzer, F.; Ahr, M. Org. Lett. 2005, 7, 1943-1946. doi:10.1021/ol050340v

21. Knochel, P., Ed. Handbook of Functionalized Organometallics; Wiley-VCH: Weinheim, Germany, 2005. doi:10.1002/9783527619467

22. Yoshida, J. Flash Chemistry: Fast Organic Synthesis in Microsystems; Wiley: Hoboken, N.J., 2008.

23. Fletcher, P. D. I.; Haswell, S. J.; Pombo-Villar, E.; Warrington, B. H.; Watts, P.; Wong, S. Y. F.; Zhang, X. Tetrahedron 2002, 58, 4735-4757. doi:10.1016/S0040-4020(02)00432-5

24. Jähnisch, K.; Hessel, V.; Löwe, H.; Baerns, M. Angew. Chem., Int. Ed. 2004, 43, 406-446. doi:10.1002/anie.200300577

25. Yoshida, J. Chem. Commun. 2005, 4509-4516. doi:10.1039/b508341a

26. Doku, G. N.; Verboom, W.; Reinhoudt, D. N.; van den Berg, A. Tetrahedron 2005, 61, 2733-2742. doi:10.1016/j.tet.2005.01.028

27. Yoshida, J.; Nagaki, A.; Iwasaki, T.; Suga, S. Chem. Eng. Technol. 2005, 28, 259-266. doi:10.1002/ceat.200407127

28. Geyer, K.; Codee, J. D. C.; Seeberger, P. H. Chem.-Eur. J. 2006, 12, 8434-8442. doi:10.1002/chem.200600596

29. Whitesides, G. Nature 2006, 442, 368-373. doi: $10.1038 /$ nature05058

30. deMello, A. J. Nature 2006, 442, 394-402. doi:10.1038/nature05062

31. Song, H.; Chen, D. L.; Ismagilov, R. F. Angew. Chem., Int. Ed. 2006, 45, 7336-7356. doi:10.1002/anie.200601554

32. Kobayashi, J.; Mori, Y.; Kobayashi, S. Chem.-Asian J. 2006, 1, 22-35. doi:10.1002/asia.200600058

33. Mason, B. P.; Price, K. E.; Steinbacher, J. L.; Bogdan, A. R.; McQuade, D. T. Chem. Rev. 2007, 107, 2300-2318. doi:10.1021/cr050944c

34. Ahmed-Omer, B.; Brandtand, J. C.; Wirth, T. Org. Biomol. Chem. 2007, 5, 733-740. doi:10.1039/b615072a

35. Watts, P.; Wiles, C. Chem. Commun. 2007, 443-467. doi:10.1039/b609428g

36. Yoshida, J.; Nagaki, A.; Yamada, T. Chem.-Eur. J. 2008, 14, 7450-7459. doi:10.1002/chem.200800582

37. Fukuyama, T.; Rahman, M. T.; Sato, M.; Ryu, I. Synlett 2008, 151-163. doi:10.1055/s-2007-1000884

38. McMullen, J. P.; Jensen, K. F. Annu. Rev. Anal. Chem. 2010, 3, 19-42. doi:10.1146/annurev.anchem.111808.073718
39. Yoshida, J. Chem. Rec. 2010, 10, 332-341. doi:10.1002/tcr.201000020

40. Yoshida, J.; Kim, H.; Nagaki, A. ChemSusChem 2011, 4, 331-340. doi:10.1002/cssc.201000271

41. Watts, P.; Wiles, C.; Haswell, S. J.; Pombo-Villar, E.; Styring, P. Chem. Commun. 2001, 990-991. doi:10.1039/b102125g

42. Suga, S.; Okajima, M.; Fujiwara, K.; Yoshida, J. J. Am. Chem. Soc. 2001, 123, 7941-7942. doi:10.1021/ja015823i

43. Fukuyama, T.; Shinmen, M.; Nishitani, S.; Sato, M.; Ryu, I. Org. Lett. 2002, 4, 1691-1694. doi:10.1021/ol0257732

44. Ueno, M.; Hisamoto, H.; Kitamori, T.; Kobayashi, S. Chem. Commun. 2003, 936-937. doi:10.1039/b301638b

45. Garcia-Egido, E.; Spikmans, V.; Wong, S. Y. F.; Warrington, B. H. Lab Chip 2003, 3, 73-76. doi:10.1039/b302381h

46. Lai, S. M.; Martin-Aranda, R.; Yeung, K. L. Chem. Commun. 2003, 218-219. doi:10.1039/b209297b

47. Mikami, K.; Yamanaka, M.; Islam, M. N.; Kudo, K.; Seino, N.; Shinoda, M. Tetrahedron Lett. 2003, 44, 7545-7548. doi:10.1016/S0040-4039(03)01835-5

48. Kobayashi, J.; Mori, Y.; Okamoto, K.; Akiyama, R.; Ueno, M.; Kitamori, T.; Kobayashi, S. Science 2004, 304, 1305-1308. doi:10.1126/science.1096956

49. Nagaki, A.; Kawamura, K.; Suga, S.; Ando, T.; Sawamoto, M.; Yoshida, J. J. Am. Chem. Soc. 2004, 126, 14702-14703. doi:10.1021/ja044879k

50. Lee, C.-C.; Sui, G.; Elizarov, A.; Shu, C. J.; Shin, Y.-S.; Dooley, A. N.; Huang, J.; Daridon, A.; Wyatt, P.; Stout, D.; Kolb, H. C.; Witte, O. N.; Satyamurthy, N.; Heath, J. R.; Phelps, M. E.; Quake, S. R.; Tseng, H.-R. Science 2005, 310, 1793-1796. doi:10.1126/science.1118919

51. Horcajada, R.; Okajima, M.; Suga, S.; Yoshida, J. Chem. Commun. 2005, 1303-1305. doi:10.1039/b417388k

52. Kawaguchi, T.; Miyata, H.; Ataka, K.; Mae, K.; Yoshida, J. Angew. Chem., Int. Ed. 2005, 44, 2413-2416. doi:10.1002/anie.200462466

53. Ducry, L.; Roberge, D. M. Angew. Chem., Int. Ed. 2005, 44, 7972-7975. doi:10.1002/anie.200502387

54. Nagaki, A.; Togai, M.; Suga, S.; Aoki, N.; Mae, K.; Yoshida, J. J. Am. Chem. Soc. 2005, 127, 11666-11675. doi:10.1021/ja0527424

55. He, P.; Watts, P.; Marken, F.; Haswell, S. J. Angew. Chem., Int. Ed. 2006, 45, 4146-4149. doi:10.1002/anie.200600951

56. Uozumi, Y.; Yamada, Y.; Beppu, T.; Fukuyama, N.; Ueno, M.; Kitamori, T. J. Am. Chem. Soc. 2006, 128, 15994-15995. doi:10.1021/ja066697r

57. Tanaka, K.; Motomatsu, S.; Koyama, K.; Tanaka, S.; Fukase, K. Org. Lett. 2007, 9, 299-302. doi:10.1021/ol062777o

58. Sahoo, H. R.; Kralj, J. G.; Jensen, K. F. Angew. Chem., Int. Ed. 2007, 46, 5704-5708. doi:10.1002/anie.200701434

59. Hornung, C. H.; Mackley, M. R.; Baxendale, I. R.; Ley, S. V. Org. Process Res. Dev. 2007, 11, 399-405. doi:10.1021/op700015f

60. Iwasaki, T.; Nagaki, A.; Yoshida, J. Chem. Commun. 2007, 1263-1265. doi:10.1039/b615159k

61. Nagaki, A.; Iwasaki, T.; Kawamura, K.; Yamada, D.; Suga, S.; Ando, T.; Sawamoto, M.; Yoshida, J. Chem.-Asian J. 2008, 3 1558-1567. doi:10.1002/asia.200800081

62. Nagaki, A.; Tomida, Y.; Yoshida, J. Macromolecules 2008, 41, 6322-6330. doi:10.1021/ma800769n

63. Fukuyama, T.; Kobayashi, M.; Rahman, M. T.; Kamata, N.; Ryu, I. Org. Lett. 2008, 10, 533-536. doi:10.1021/ol702718z 
64. Nagaki, A.; Tomida, Y.; Miyazaki, A.; Yoshida, J. Macromolecules 2009, 42, 4384-4387. doi:10.1021/ma900551a

65. Tricotet, T.; O'Shea, D. F. Chem.-Eur. J. 2010, 16, 6678-6686. doi:10.1002/chem.200903284

66. Greenway, G. M. S.; Haswell, J.; Morgan, D. O.; Skelton, V.; Styring, P. Sens. Actuators, B 2000, 63, 153-158. doi:10.1016/S0925-4005(00)00352-X

67. Haswell, S. J.; O'Sullivan, B.; Styring, P. Lab Chip 2001, 1, 164-166. doi:10.1039/b104035a

68. Niwa, S.; Eswaramoorthy, M.; Nair, J.; Raj, A.; Itoh, N.; Shoji, H.; Namba, T.; Mizukami, F. Science 2002, 295, 105-107. doi:10.1126/science.1066527

69. Jas, G.; Kirschning, A. Chem.-Eur. J. 2003, 9, 5708-5723. doi:10.1002/chem.200305212

70. Basheer, C.; Hussain, F. S. J.; Lee, H. K.; Valiyaveettil, S. Tetrahedron Lett. 2004, 45, 7297-7300. doi:10.1016/j.tetlet.2004.08.017

71. Liu, S.; Fukuyama, T.; Sato, M.; Ryu, I. Org. Process Res. Dev. 2004, 8, 477-481. doi:10.1021/op034200h

72. Kunz, U.; Schönfeld, H.; Solodenko, W.; Jas, G.; Kirschning, A. Ind. Eng. Chem. Res. 2005, 44, 8458-8467. doi:10.1021/ie048891x

73. Comer, E.; Organ, M. G. J. Am. Chem. Soc. 2005, 127, 8160-8167. doi:10.1021/ja0512069

74. Lee, C. K. Y.; Holmes, A. B.; Ley, S. V.; McConvey, I. F.; Al-Duri, B.; Leeke, G. A.; Santos, R. C. D.; Sevilled, J. P. K. Chem. Commun. 2005, 2175-2177. doi:10.1039/b418669a

75. Mauger, C.; Buisine, O.; Caravieilhes, S.; Mignani, G. J. Organomet. Chem. 2005, 690, 3627-3629. doi:10.1016/j.jorganchem.2005.03.071

76. Kirschning, A.; Solodenko, W.; Mennecke, K. Chem.-Eur. J. 2006, 12, 5972-5990. doi:10.1002/chem.200600236

77. Shore, G.; Morin, S.; Organ, M. G. Angew. Chem., Int. Ed. 2006, 45, 2761-2766. doi:10.1002/anie.200503600

78. Shi, G.; Hong, F.; Liang, Q.; Fang, H.; Nelson, S.; Weber, S. G. Anal. Chem. 2006, 78, 1972-1979. doi:10.1021/ac051844+

79. Rahman, M. T.; Fukuyama, T.; Kamata, N.; Sato, M.; Ryu, I. Chem. Commun. 2006, 2236-2238. doi:10.1039/b600970k

80. Murphy, E. R.; Martinelli, J. R.; Zaborenko, N.; Buchwald, S. L.; Jensen, K. F. Angew. Chem., Int. Ed. 2007, 46, 1734-1737. doi:10.1002/anie.200604175

81. Kawanami, H.; Matsushima, K.; Sato, M.; Ikushima, Y. Angew. Chem., Int. Ed. 2007, 46, 5129-5132. doi:10.1002/anie.200700611

82. Yamada, Y. M. A.; Watanabe, T.; Torii, K.; Uozumi, Y. Chem. Commun. 2009, 5594-5596. doi:10.1039/b912696a

83. Jin, J.; Cai, M.-M.; Li, J. Synlett 2009, 2534-2538. doi:10.1055/s-0029-1217730

84. Ahmed-Omer, B.; Barrow, D. A.; Wirth, T. Tetrahedron Lett. 2009, 50, 3352-3355. doi:10.1016/j.tetlet.2009.02.133

85. McMullen, J. P.; Stone, M. T.; Buchwald, S. L.; Jensen, K. F. Angew. Chem., Int. Ed. 2010, 49, 7076-7080. doi:10.1002/anie.201002590

86. Usutani, H.; Tomida, Y.; Nagaki, A.; Okamoto, H.; Nokami, T.; Yoshida, J. J. Am. Chem. Soc. 2007, 129, 3046-3047. doi:10.1021/ja068330s

87. Nagaki, A.; Tomida, Y.; Usutani, H.; Kim, H.; Takabayashi, N.; Nokami, T.; Okamoto, H.; Yoshida, J. Chem.-Asian J. 2007, 2, 1513-1523. doi:10.1002/asia.200700231

88. Nagaki, A.; Takabayashi, N.; Tomida, Y.; Yoshida, J. Org. Lett. 2008, 10, 3937-3940. doi:10.1021/ol8015572
89. Nagaki, A.; Kim, H.; Yoshida, J. Angew. Chem., Int. Ed. 2008, 47, 7833-7836. doi:10.1002/anie.200803205

90. Nagaki, A.; Takizawa, E.; Yoshida, J. J. Am. Chem. Soc. 2009, 131, 1654-1655. doi:10.1021/ja809325a

91. Nagaki, A.; Takabayashi, N.; Tomida, Y.; Yoshida, J. Beilstein J. Org. Chem. 2009, 5, No. 16. doi:10.3762/bjoc.5.16

92. Tomida, Y.; Nagaki, A.; Yoshida, J. Org. Lett. 2009, 11, 3614-3617. doi:10.1021/ol901352t

93. Nagaki, A.; Kim, H.; Yoshida, J. Angew. Chem., Int. Ed. 2009, 48, 8063-8065. doi:10.1002/anie.200904316

94. Nagaki, A.; Takizawa, E.; Yoshida, J. Chem.-Eur. J. 2010, 16, 14149-14158. doi:10.1002/chem.201000815

95. Nagaki, A.; Kim, H.; Moriwaki, Y.; Matsuo, C.; Yoshida, J. Chem.-Eur. J. 2010, 16, 11167-11177. doi:10.1002/chem.201000876

96. Nagaki, A.; Kim, H.; Matsuo, C.; Yoshida, J. Org. Biomol. Chem. 2010, 8, 1212-1217. doi:10.1039/b919325c

97. Tomida, Y.; Nagaki, A.; Yoshida, J. J. Am. Chem. Soc. 2011, 133, 3744-3747. doi:10.1021/ja110898s

98. Kim, H.; Nagaki, A.; Yoshida, J. Nat. Commun. 2011, 2, No. 264. doi:10.1038/ncomms1264

99. Suga, S.; Yamada, D.; Yoshida, J. Chem. Lett. 2010, 39, 404-406. doi:10.1246/cl.2010.404

100.Nagaki, A.; Kenmoku, A.; Moriwaki, Y.; Hayashi, A.; Yoshida, J. Angew. Chem., Int. Ed. 2010, 49, 7543-7547. doi:10.1002/anie.201002763

\section{License and Terms}

This is an Open Access article under the terms of the Creative Commons Attribution License (http://creativecommons.org/licenses/by/2.0), which permits unrestricted use, distribution, and reproduction in any medium, provided the original work is properly cited.

The license is subject to the Beilstein Journal of Organic Chemistry terms and conditions:

(http://www.beilstein-journals.org/bjoc)

The definitive version of this article is the electronic one which can be found at: doi: $10.3762 /$ bjoc 7.122 